\title{
NOTICES INDUSTRIELLES
}

\section{Pour compléter la Documentation de la Houille Blanche, nous pensons être agréables} à nos Lecteurs en leur donnant, sous cette rubrique, des informations industrielles, financières, commerciales, susceptibles de leur rendre des services immédiats.

\section{Groupes électrogènes de secours " Force et Lumière " pour stations hydroélectriques et usages industriels.}

\begin{abstract}
Par R. VAN MUYDEN, Ingénieur, Membre sociélaire de la Société des Ingénienrs civils de France.
Le manque d'eau, cette année, sur de nombreux points de France et dans les Alpes particulièrement, a provoqué des crises de chómage, conséquence logique des pannes et restrictions dont a souffert la production industrielle de la force motrice. Le moyen de parer efficacement de ces difficultés nous est donné dans l'article ci-dessous dont la primeùr a été réservée à nos lecteurs, et que nous devons à lobligeance de M. Van Muyden, Ingénieur, membre sociétarre de la Société des Ingénieurs clvils de France.
\end{abstract}

Les stations hydro-électriques des Alpes manquent d'eau en hiver.

Celles du Jura sont en mauvaise posture l'été,

Dans la région parisienne en hiver par suite des pointes, les industriels souffrent de pannes et de restrictions très ennuyeuses pour la production.

Ce sont ces faits qui ont engagé les Etablissements Japy Frères: et $\mathrm{C}^{\mathrm{ie}}$, à Beaucourt (Territoire de Belfort) à établir des groupes électrogènes de moyenne puissance marchant à l'essence, au benzol, ćventuellement naphtaline ou semi-Diesel ou Diesel.

La caractéristique essentielle d'un groupe électrogène de secours est une mise en marche immédiate.

Dès que le courant du secteur vient à manquer, il faut que le groupe électrogène assure le service de suite.

Nous verrons plus loin comment le constructeur des moteurs de secours a résolu élégamment ce problème des plus délicats.

Les Ateliers de Construction de matériel électrique "Japy frères et $\mathrm{C}^{\mathrm{ie}}$ "reçoivent leur courant de la station hydro-électrique du Refrain située dans le Jura.

Par suite de la sécheresse de cet été, des pannes fréquentes eurent lieu et cela engagea la Direction à étudier un groupe de secours à installer dans ses ateliers.

C'est ce dernier que nous allon's décrire succinctement.

\section{LEURS AVANTAGES}

\section{Econome D'Installation}

Fondations peu importantes, installation immédiate, encombrement réduit, transport facile, suppression des chaudières, gazogènes et de toute machine auxiliaire.

\section{Économie d'Entretien}

Graissage limité au simple remplissage d'un réservoir, simplicité et sécurité du service, main d'œuvre limitée à une simple surveillance.

\section{ECONOMTE DE TEMPS.}

Mise en route instantanće.

\section{VITESSE CONSTANTE}

Un régulateur spécial maintient une vitesse rigoureusement constante permettant de produire directement l'éclairage électrique même pour des charges très variables.

\section{Interchangeabilité des Piéces}

Permettant le remplacement d'une pièce ou d'un organe quelconque sans délai ni retouche.

\section{LEURS APPLICATIONS PRINCIPALES}

Groupes électrogènes d'appoint, pour fournir la force motrice et l'éclairage d'ateliers, magasins, usines, chantiers, etc...

Stations de pompage pour usines et chantiers.

En raison de leur prix réduit, de leur faible encombrement et de la mise en marche instantanée, ces moteurs sont particulière ment recherchés comme :

Groupes électrogdnés de secours pour parer immédiatement aux pannes de secteur ou de la force motrice.

Groupes moto-pompes de secours permettant d'évacuer l'eau en cas d'inondation sanš être à la merci du courant électrique.

$$
\text { I }
$$

Caractéristiques du Moteur "Renault " à essence ou benzol, de 100 C. V. effectifs, du type "accouplement direct ", avec alternateur triphasé.

Ils sont particulièrement utilisés comme groupes électrogènes de secours pour petites stations hydro-électriques, car ils sont mis en marche presque instantanément.

IIs sont plus particulièrement désignés comme groupes de secours ou d'appoint pour fonctionnement intermittent.

Ils se composent essentiellement du moteur proprement dit, d'un socle, d'un volant et d'un manchon d'entrainement.

Le moteur est à 8 cylindres disposés en $\mathrm{V}$, de façon à procurer un encombrement aussi réduit que possible.

$$
\begin{aligned}
& \text { Alésage } \ldots \ldots \ldots \ldots \ldots \ldots \ldots \ldots 125 \mathrm{~m} / \mathrm{m} \\
& \text { Course } \ldots \ldots \ldots \ldots \ldots \ldots \ldots \ldots \ldots, 150 \mathrm{~m} / \mathrm{m} \\
& \text { Vitesse de régime . . . . . . . . . . } 800 / 1200 \mathrm{t} / \mathrm{m}
\end{aligned}
$$

Cylindres. - Les cylindres sont en acier pris dans la masse et jumelés par groupes de deux.

Chaque groupe est enveloppé dans une même chemise en tôle d'acier.

Ils reposent sur un carter supérieur en aluminium.

Le vilbrequin en acier estampé est porté par deux paliers extrêmes et une portée centralè.

Les bielles en acier spécial estampé à section' double 'T sont articulées autour de l'axe des pistons.

Les pistons sont en fonte et traversés par des axes immobilisés.

Distribution. - Les soupapes placées de part et d'autre des deux rangées de cylindres sont commandées par deux arbres de distribution placés à la partie supérieure des cylindres.

Les arbres de distribution' sont commandés par l'intermédiaire de deux arbres inclinés recevant leur mouvement d'un pignon calé sur le vilbrequin. 
Allumage. - L'allumage est double et indépendant; il se fait au moyen de quatre magnétos à haute tension. Elles sont commandées par des pignons à denture hélicoïdale.

Mise en marche. - La mise en marche instantanée s'effectue au moyen d'un démarreur spécial fourni avec le moteur.

Les deux carburateurs du type "Renault "sont automatiques à tous les régimes.

\section{CONSOMMATIONS HORAIRES}

\begin{tabular}{c|c|c|c|c} 
Poids en & Nombre de & Consommations horaires \\
kgs env. & tours & Puissance & essence & huile \\
& - & - & $34 l$. & \\
640 & 800 & $90 \mathrm{HP}$ & 36 l. & \\
& 1000 & $105 \mathrm{HP}$ & 37.5 & 2 à 5 litres
\end{tabular}

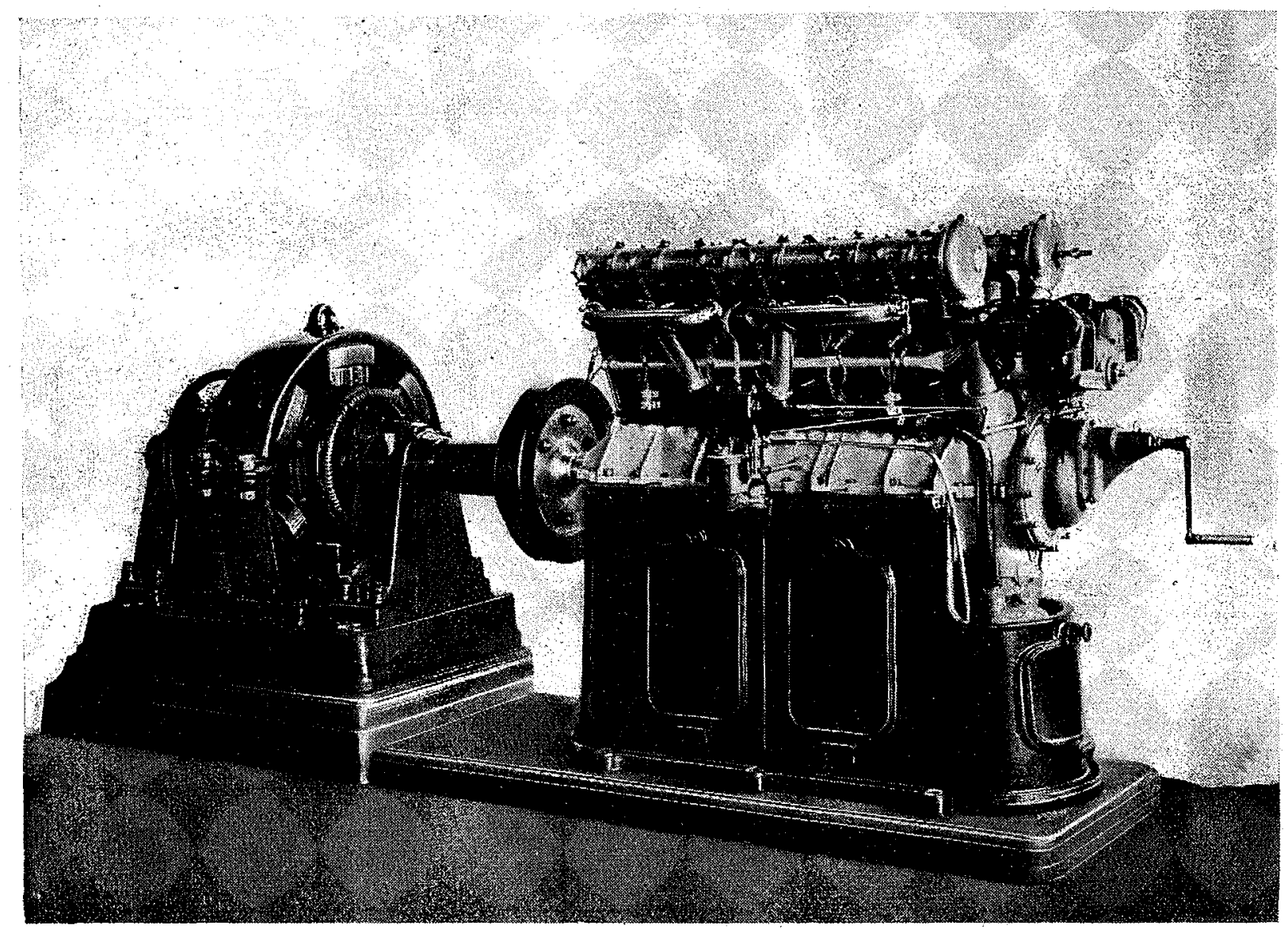

Moteur industriel Renault, à essence ou benzol, 12 cylindres en. V.

800 tours par minute. $145 \mathrm{HP}$ effectifs. -1.000 tours par minute, $180 \mathrm{HP}$ effectifs. -1.200 tours par minute, $200 \mathrm{HP}$ effectifs Alésage $125 \mathrm{~m} / \mathrm{m}$. - Course $150 \mathrm{~m} / \mathrm{m}$.

Régulation. - Le régulateur centrifuge est monté sur l'arbre du vilbrequin et agit directement sur l'admission des gaz au carburateur. Il maintient la vitesse constante.

Refroidissement. - Il s'effectue par circulation d'eau sous pression au moyen de pompe centrifuge à double refoulement. Un réservoir de 2000 litres est prévu à cet effet.

Graissage. - Il est assuré par circulation d'huile sous pression au moyen d'une pompe à engrenages logés dans le fond du carter. Le carter inférieur forme réservoir. Cette pompe à engrenages comprenant deux étages, le corps inférieur plus petit reçoit l'huile fraíche du réservoir et la refoule dans le fond du carter servant ainsi à compenser les pertes.

Cette disposition permet d'introduire de l'huile dans le carter pendant la marche industrielle.

Socle. - Le bâti en fonte avec portes de visite forme socle.

Volant. - Le volant en acier coulé est monté à emmanchement conique sur l'extrémité arrière du vilbrequin.

Manchon d'entraînement. - Le manchon d'entraînement est en acier coulé. Il est fourni avec les doigts et les bagues de caoutchouc.

Encombrement. - Les cotes d'encombrement sont les suivantes :

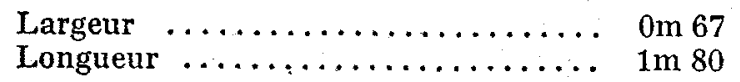

Le moteur est livré prêt à une mise en marche industrielle (carburateurs, magnétos, volant, socle, tuyauterie d'eau réunissant la pompe aux cylindres, coudes d'échappement).

Les silencieux, réservoirs d'eau, d'essence et d'huile sont liviés à part ainsi que la tuyauterie extérieure.

\section{II}

Caractéristiques de l'Alternateur a Japy série L, V. M, 1921

L'Alternateur synchrone à deux paliers pour courant alternatif triphasé type C. T. 84 ter, est construit pour les données de mache suivantes :

a) Puissance utile disponible en marche "continue " sous $\cos 0=1 \ldots \ldots \ldots \ldots \ldots \ldots \ldots \ldots \ldots \ldots \ldots, 55 \mathrm{KW}$

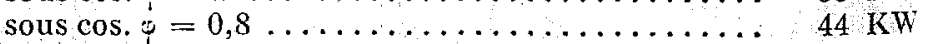

b) Puissance absorbée en HP (excitation comprise) sous cos. $\psi=1 \ldots \ldots \ldots \ldots \ldots \ldots \ldots \ldots \ldots$. 85

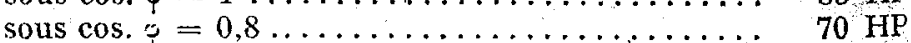

c) Puissance d'excitation nécessaire (maximum prévu pour $25 \%$ de surcharge).

sous $\cos \varphi=1 \ldots \ldots \ldots \ldots \ldots \ldots \ldots \ldots \ldots$ watts

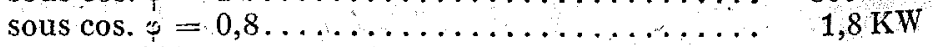

d) Vitesse 1000 tours par minute.

e) Fréquence : 50 périodes par seconde.

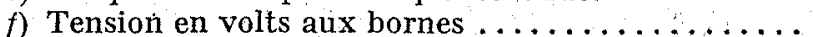
( 4 e borne pour le point neutre).

Cette machine est établie de façon à pouvoir alimenter un tetseau triphasé " Force et Lumière ".

La tension de 220 volts correspond à la tension entre bornes de l'alternateur bobiné en étoile; celle de 125 volts est celle mesuréé entre l'une de ces mêmes bormes et le point neutre.

Les moteurs installés sur le réseau peuvent donc être alimentés sous une tension de 200 à 210 volts environ.

L'éclairage (réseau à 4 fils) est alimenté sous une tension de $110 / 115$ volts environ sans aucune transformation. 


\section{DESCRIPTION}

Disposition à deux paliers avec coussinets en bronze "qualité spéciale a à grande surface de frottement du type à graissage automatique au moyen de bagues.

Inducteur tournant à 6 pôles; bâti forme ronde en fonte douce modèle " ouvert".

Stator. - Le noyau induit est constitué par des tôles minces de haute perméabilité et de faible coefficient d'hystérésis soigneu-
Arbre. - L'arbre est en acier Siemens Martin lorgé et prolongé à ses deux extrémités. L'une des extrémités est destinée à recevoir la poulie ou manchon d'accouplement avec la machine motrice, tandis que l'excitatrice est montée en bout d'arbre.

L'arbre porte également les bagues collectrices en bronze spécial ; ces dernières sont frettées à chaud sur l'arbre et isolées. de celui-ci au moyen de micanite.

Les porte-balais sont du type "coulissant ", à ressort de pression réglables, modèle "Marine ".

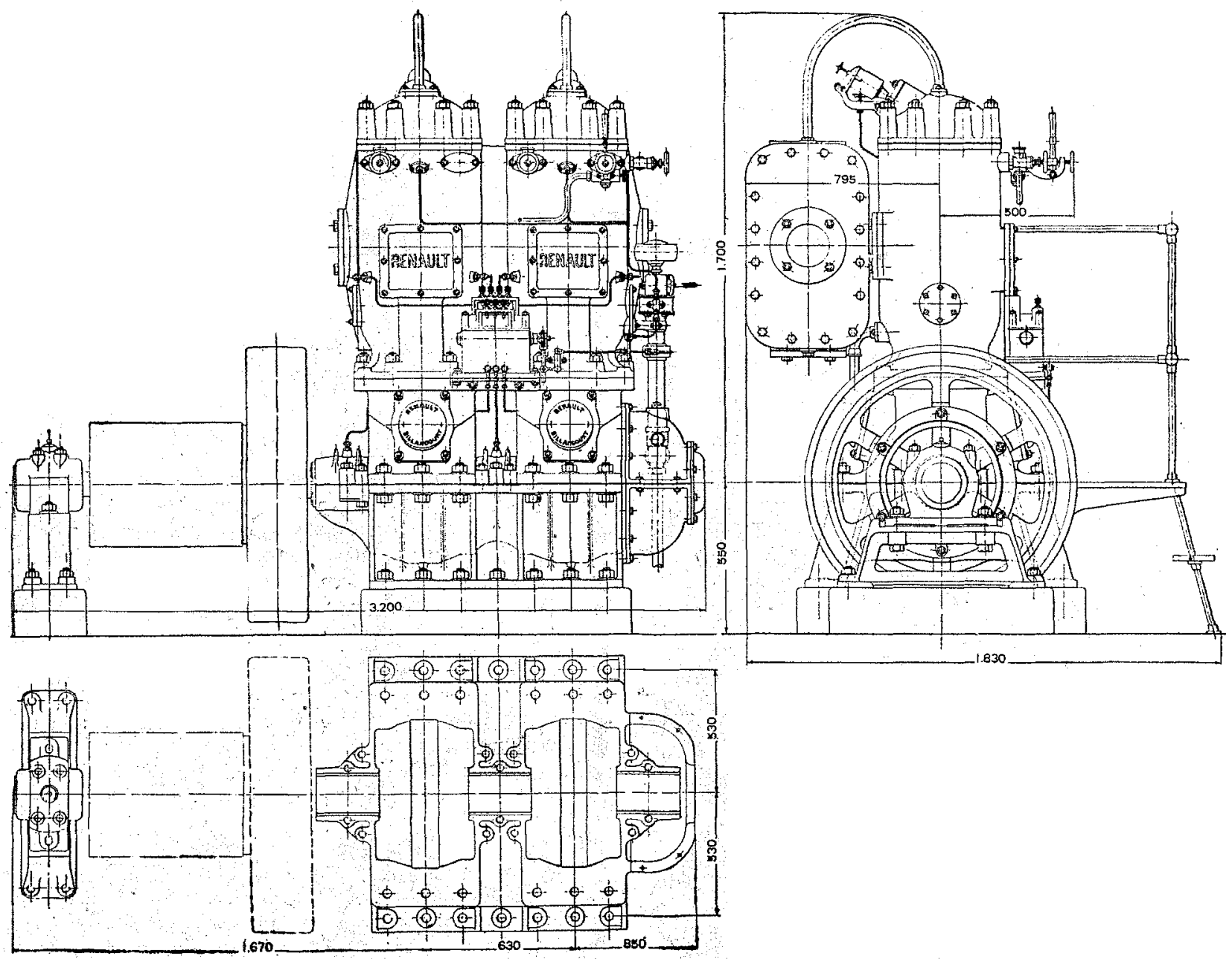

Moteur industriel Renault a huile lourde, de 90 C.V. effectifs, a 325 tours par minute, type Semi-Diesel.

sement isolées dans le but de réduire au minimum les pertes par courants de Foucault.

Ces tôles sont fixées dans les nervures d'une carcasse en fonte et maintenues solidement à I'aide de deux flasques clavetées.

Assemblées en paquets, elles sont supportées par des cloisons laissant un passage d'air suffisant pour assurer un refroidissement efficace du noyau.

Le bobinage induit, fait a la main, a enroulement ondule is est logé dans des encoches semi-fermées et isolées dè la masse au moyen d'isolants spéciaux ; les développantes sont soigneusement enrubannées à l'aide de rubans de toile huilée.

Les 3 phases de l'enroulement induit sont connectées en étoile et reliées aux trois bornes de départ.

Sur demande, il est possible d’ajouter une quatrième borne pour le point neutre.

Rotor. - Le rotor est constitue par un tambour monobloc en acier demi-dúr forgé. Les pièces polaires sont lamellées et formées de tôles bien assemblées et soigneusement rivées.

Les pôles sont fixés sur la roue polaire par des boulons d'acier spécial à grande résistance vissés dảns des clavettes en acier forgé.

Les bobines inductrices sont constituées par des fils de cuivre isolé.

Les spires sont isalées les unes des autres par du carton comprimé imprégné de vernis isolant.

Toute la bobine est ensuite soumise à chaud à une imprégnation par compoundage. La bobine devient un corps rigide et compact pouvant assurer un service industriel intensif.

Le moment d'inertie "PD2" de la partie tournante est de 15 kilogrammètres carrés.
Excitatrice. - L'excitatrice à courant continu est du type "ouvert ", tension variable de 40 à 100 volts.

Elle est accouplée en bout d'arbre par manchon semi-élastique et montée sur socle fonte reliant l'alternateur et l'excitatrice (voir dessin $\left.n^{\circ} 4974\right)$.

Cette disposition permet le clémontage facile, soit de l'excitatrice, soit de l'alternateur, sans qu'il soit nécessaire de démonter les deux machines, comme on est obligé de le faire quand l'excitatrice est directement montée en bout d'arbre de l'alternateur sans interposition de manchon d'accouplement.

Ventilation. - Une attention particulière a été apportée a la ventilation efficace de toutes les parties actives de l'alternateur de façon a ce que toutes les parties engendrant de la chaleur soient énergiquement balayées par de l'air pur.

Des ailettes de ventilation à action puissante sont fixées des deux côtés de la roue polaire; elles aspirent l'air axialement et le refoulent en partie contre les têtes des bobines de l'induit et en partie entre les pôles.

Après avoir balayé les bobines inductrices et l'enroulement induit, l'air traverse le fer de l'induit par des canaux de ventilation largement dimensionnés et arrive a la carcasse d'où il s'échappe par des orifices de ventilation.

\section{III}

\section{Garańties. - Conditions générales de fonciionnement,}

Les enroulements de l'alternateur et de l'excitatrice sont soigneusement étuvés dans le vide avant imprégnation ; toute trace 
d'humidité disparue, les enroulements sont imprégnés de vernis isolant au moyen d'un dispositif spécial par vide et pression.

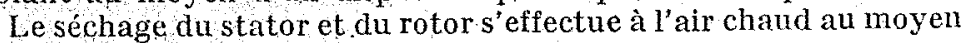
de batteries de séchage spéciales.

Le matériel répond entièrement, au point de vue techniçue, aux prescriptions et spécifications des règles françaises d'unification du matériel électrique établies par le Comité Electrotechnique francais et adoptées par l'Union des. Syndicats de l'Electricité (édition juin 1919).

\section{RENDEMENTS}

a) Rendement à $1 / 1$ charge en $\%$.

sous cos. $\vdots=1 . \ldots \ldots \ldots \ldots \ldots \ldots \ldots \ldots \ldots \ldots .91 \%$ sous cos. $=0,8 \ldots \ldots \ldots \ldots \ldots \ldots \ldots \ldots \ldots . \ldots \ldots$

b) Surélévation de tension ì 1/1 charge.

En coupant brusquement le courant débité par la machine à $1 / 1$ charge, la vitesse et l'excitation étant constantes, l'élévation de tension est de

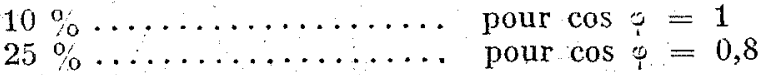

\section{c) Echauffement :}

La température mesurée par thermomètre placé sur les enroule. ments et les pôles, à l'arrêt, ne dépasse pas plus de $45^{\circ}$ la tempéra ture ambiante après une marche continue de 4 heures à $1 / 1$ charge d) Surcharge

Sans fonctionnement défectueux, la machíne peut supporter une surcharge de $25 \%$ pendant une demi-heure.

c) Isolement :

La tension d'essai à la masse est de 2.000 volts.

\section{Rhéostats}

Les résistances sont renfermées dans un châssis en fonte dans lequel l'air de la ventilation circule facilement.

Ils se distinguent avantageusement par leur construction solide et par leur ventilation efficace due à la disposition appropriée des éléments de résistance.

\section{Tableaux de Distribution et de Manouvre}

Il est fourni également sur demande des tableaux complets montés sur cadre en fer cornière et portant les appareils et ćventuellement les départs nécessaires.

\section{Caractéristiques du Moteur " Renault " à huile lourde, modèle commandé par courroie type ( semi-diesel ).}

Ils sont surtout employés dans les centrales hydro-électriques de moyenne puissance et usage industriel, devant assurer un fonctionnement intensif.

Ce type est particulièrement intéressant par suite de sa faible consommation, de son extrême simplicité et de sa grande robustesse.

Il convient fort bien pour la commande de génératrices courant continu (Centrales de tramway) et d'alternateurs, par suite de l'ordre de grandeur de sa vitesse.

Louis Renault, le constructeur bien connu, créateur des tanks de la victoire, construit des a Demi-Diesel " type "L. R. " de 40 à $300 \mathrm{C}$. V. eff. Le moteur Diesel, construction « L. Renault „ $300 \mathrm{HP}$ type "Marine " tourne à 500 tours par minute. De nombreux spécimens sont en fonctionnement industriel depuis quelques années.

Le type spécial "Marine ", des Etablissements «Renault " obtient présentement un très grand succès dans la marine marchande française ef alliée.

Nous allons décrire succinctement le type classique à huile lourde de $80 \mathrm{HP}$ en service continu capable d'une surcharge. de $15 \%$ momentanément, vitesse 325 tours par minute.

Au point de vue général, les moteurs Renault à huile lourde présentent dans leur construction l'aspect de tous les bons moteurs Semi-Diesel ou à tête chaude, mais certains organes ont été particulièrement étudiẻs en vue d'obtenir un meilleur rendement et une plus grande simplicité de manœuvre, surveillance et démontage.

Ces moteurs utilisent toutes les huiles lourdes et en particulier lhuile de goudron de houille.

Mise en route à l'air comprimé.

Ils fonctionnent suivant le cycle bien connu des moteurs à deux temps semi-diesel ou à tête chaude.

Gycle. - L'air de combustion emmagasiné dans le cylindre se trouve comprimé lors de sa course ascendante par la partie supérieure du piston pendant que l'air extérieur est aspiré dans le carter étanche par la dépression produite par ladite montée. au moment ou le piston arrive à la fin de sa course, une certaine quantité de combustion où, par suite de la température de l'air et des parois, elle se vaporise et s'enflamme, cette combustion produisant une élévation de pression détermine la course motrice ascendante.

Pendant cette course, la partie postérieure du piston comprime l'air qui se trouve dans le carter étanche.

Arrivé à environ $20 \%$ de la fin de sa course déscendante, le piston découvre d'abord les lumieres d'échappement, les gaz brûlés s'échappent et leur pression diminie.

Peu après le piston découvre les lumières de balayage par lesquelles l'air comprimé dans le carter étanche pénètre à l'intérieur du cylindre où, guidé par un déflecteur ad hoc du piston, cet appareil est dirigé vers la partie haute et chasse du fait de sa pression les gaz restant.

A ce moment, le piston reprend sa course montante et le cycle recommence.
PARTICULARITÉS DIVERSES

Mise en marche. - La mise en marche a lieu au pétrole lampant. Lorsque la température de la salle est normale, c'est-à-dire à 10 ou $15^{\circ}$, le moteur, même à froid, démarre par un simple coup d'air Si la température est très basse, il suffit, avant de lancer à l'air, de réchauffer pendant quelques minutes une aiguille à l'aide de la Jampe fournie à cet effet.

Un moteur chaud démarre instantanément à l'huile lourde.

Changement de Marche (Puissance et vitesse).

Le changement de régime s'obtient en faisant varier la quantité de combustible injectée proportionnellement a la puissance ou la vitesse demandée.

Contrairement à ce qui se produit avec le réglage dit \& tout ou rien ", le dispositif de commande de réglage employé produit toujours une combustion à chaque tour, ce qui donne au moteur une marche très régulière et sans choc.

Tout le réglage peut être effectué indifféramment soit par une manette; soit par un régulateur.

La parfaite régulation permet de conduire en toute sùreté une génératrice électrique en obtenant le coefficient de régularité demandé. Le volant assure un coefficient d'irrégularité de 140.

Le régulateur centrifuge permet une variation de vitesse de $5 \%$ du nombre de tours normal, par action continue sur la pompe d'injection du combustible.

Pour obtenir encore un meilleur rendement, un dispositif permet un réglage de l'avance du balayage et de l'injection du combustible.

A pleine charge. - Aucun refroidissement de la tête par injection d'eau n'est nécessaire.

A faible charge. - Aucun réchauffage n'est utile.

Pompes a combustibles. - La conception et la construction des pompes à combustible sont telles qu'elles ne nécessitent qu'uì très faible entretien et qu'aucun déréglage ne peut avoir liet.

Malgré $l^{\prime}$ absence d’un presse-étoupe au piston plongeur, l'étanchéité est parfaite.

Graissage, - Un appareil central à débit réglable distribue visiblement l'huile aux différents point à graisser du moteur Cet appareil bien que fonctionnant automatiquement pent, le cas échéant, par exemple au départ, être actionné à la main.

En résumé, ce moteur est d'une construction simple et robuste ne possédant aucun dispositif compliqué ou délicat, et $n$ 'exigeantarcune surveillance; il peut être mis entre les mains d'un manouvre.

CARACTERISTIQUES ET CONSOMMATION HORAIRE Puissance en HP effectifs ............ \{ Norm. Nombre de tours - Minute.......... maxi..${ }_{325}$

Poids en kilogs, environ.................... 7500

Consommation de combustible a charge , $4 / 4 \ldots . . ., 250$ 10.000 calories en gram. avec $\quad 3 / 4 . . .20280$ $10 \%$ de tolérance, par C.V., $-1,1 / 2 . . ., 325$ eff. heure, $1 / 4, \ldots, \ldots+440$ 